\title{
INVERSION LINES OF PHOTOSPHERIC MAGNETIC FIELDS AND SOLAR CORONA
}

\author{
F. AXISA \\ Service d'Electronique Physique, Centre d'Études Nucléaires de Saclay, France \\ and \\ M.-J. MARTRES and C. MERCIER* \\ Solar Dept. Meudon Observatory, 92190 Meudon, France
}

\begin{abstract}
Solar Phys.). Two results were obtained recently at Meudon concerning the metric type III production by flares which both emphasize the importance of the local magnetic configuration involved.

The first point explores the possibility that the particular location of Flare Productive Sites in an active region is closely connected to the production of type III radio bursts (Axisa, 1974). It is shown that there is a significant difference between the type III productivity of flares which occur in different Flare Productive Sites, even in the same active region. Qualitative informations about the photospheric magnetic pattern support the point that the type III production is stimulated in the case the flares are located near the periphery of the facular pattern where the strength of the magnetic field is rather low at the photospheric level.

For example, Figure 1 shows the photospheric magnetic field pattern of the active center McMath region 8905 observed on July 27, 1967.
\end{abstract}

North polarity is delineated by full lines, south polarity by dotted lines, hatchings indicate the location of the flare producing sites (FPS).

- FPS 1 is located inside strong magnetic fields, on penumbra of a complex spot and was very poor in producing type III bursts.

- FPS 2 is located at the periphery of the facular region near the preceding spot: it produced many type III bursts.

From the study of the FPS's for several active regions, it was found that for FPS in such a situation as FPS 1 , among 67 flares, $14 \%$ only was accompanied by type III bursts; for FPS in such a situation as FPS 2, 60 flares appeared and $60 \%$ of them were associated with groups of type III bursts. Incidently it is to note that FPS 2 was also responsible for the electron events observed and discussed by Lin.

So it was found that the magnetic structure giving rise to a flare associated with type III bursts is very often characterised by the existence of an inversion line of longitudinal magnetic field between a spot (preceding or following) and its 'parasitic polarity'. This line appears in region of high gradient of longitudinal magnetic fields and vanishes very near weak fields outside the facular region.

The second point concerns the association between filaments and type III bursts (Mercier, 1973).

* Equipe de Recherche Associée du C.N.R.S. No. 306.

Gordon Newkirk, Jr. (ed.), Coronal Disturbances, 69-72. All Rights Reserved. Copyright (C) 1974 by the IAU. 


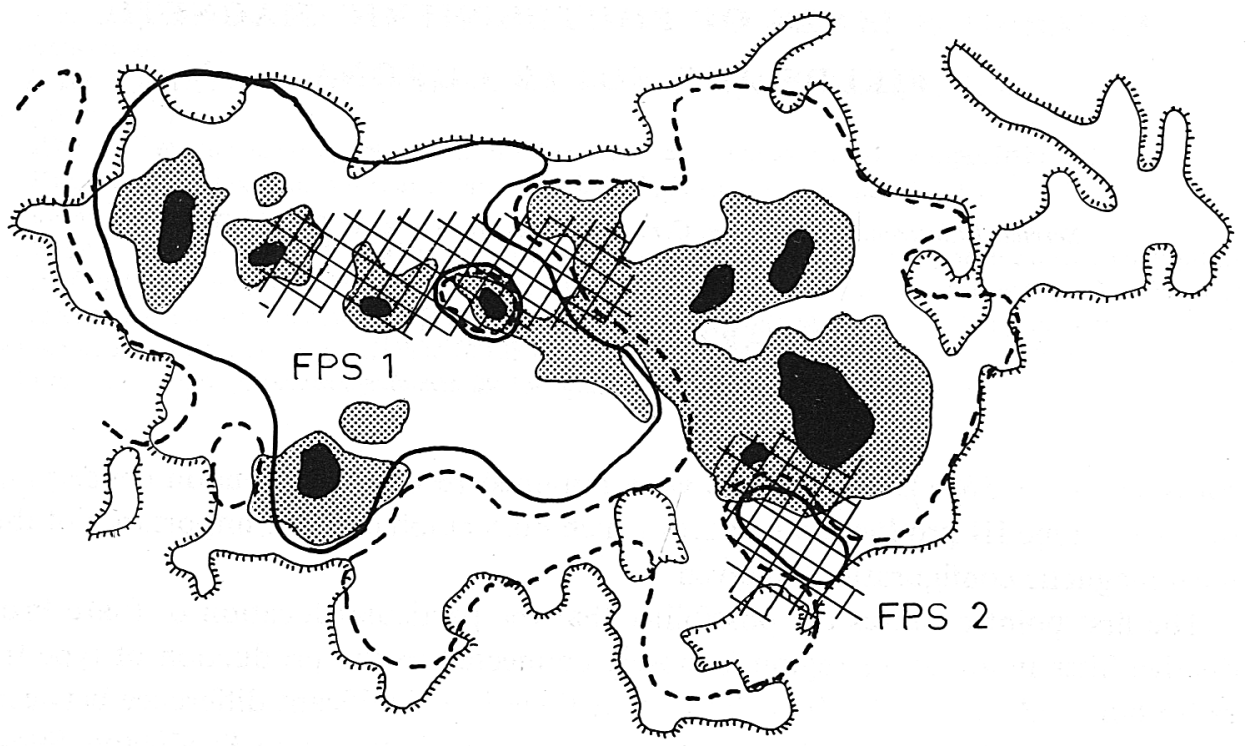

Fig. 1. Spot group McMath 8905. Observed on 1967, July 27. The spots are presented as visible on spectroheliogram $K_{1 v}$ : black areas for umbrae, and dotted for penumbrae. The facula is outlined by a 'comb'. The longitudinal magnetic polarities are indicated by a full line (north polarity) or dashed line (south polarity). Hatched regions shows the flare producing sites (FPS) of this day (from Axisa, 1973).

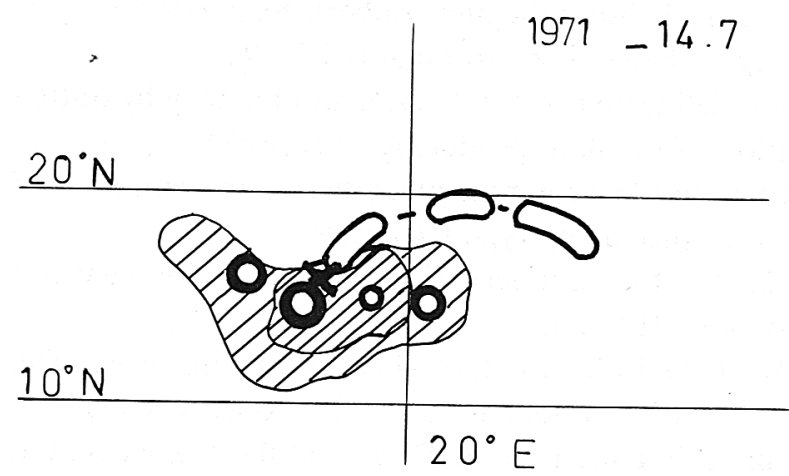

Fig. 2. Active region as seen on Synoptic Maps of the chromosphere. The facula and the spots are schematically drawn. Differences in hatching mark differences in brightness in the facula. The cross indicates the approximate position of the flare. The filament concerned appears as closed double parallel lines (from Mercier, 1973).

It is often observed that a filament (called plagefilament) appears along such an inversion line. As it is schematically shown in Figure 2, this filament is often of small size and does not last more than 3 or 4 days. Occasionally it may be directed toward a greater system of quiescent filaments.

It is worth emphasizing that the plage filament is not always present at the time of type III occurences. The delay between the type III burst and the appearance of the 
filament very rarely exceeds one day. The histogram of distribution of this delay in the observed cases is presented in Figure 3. Negative delays correspond to cases where filaments appeared after the type III burst occurence.

In addition, it is observed, in one case, that the filament becomes more visible one day after the first observation, suggesting the time scale of the formation of such a

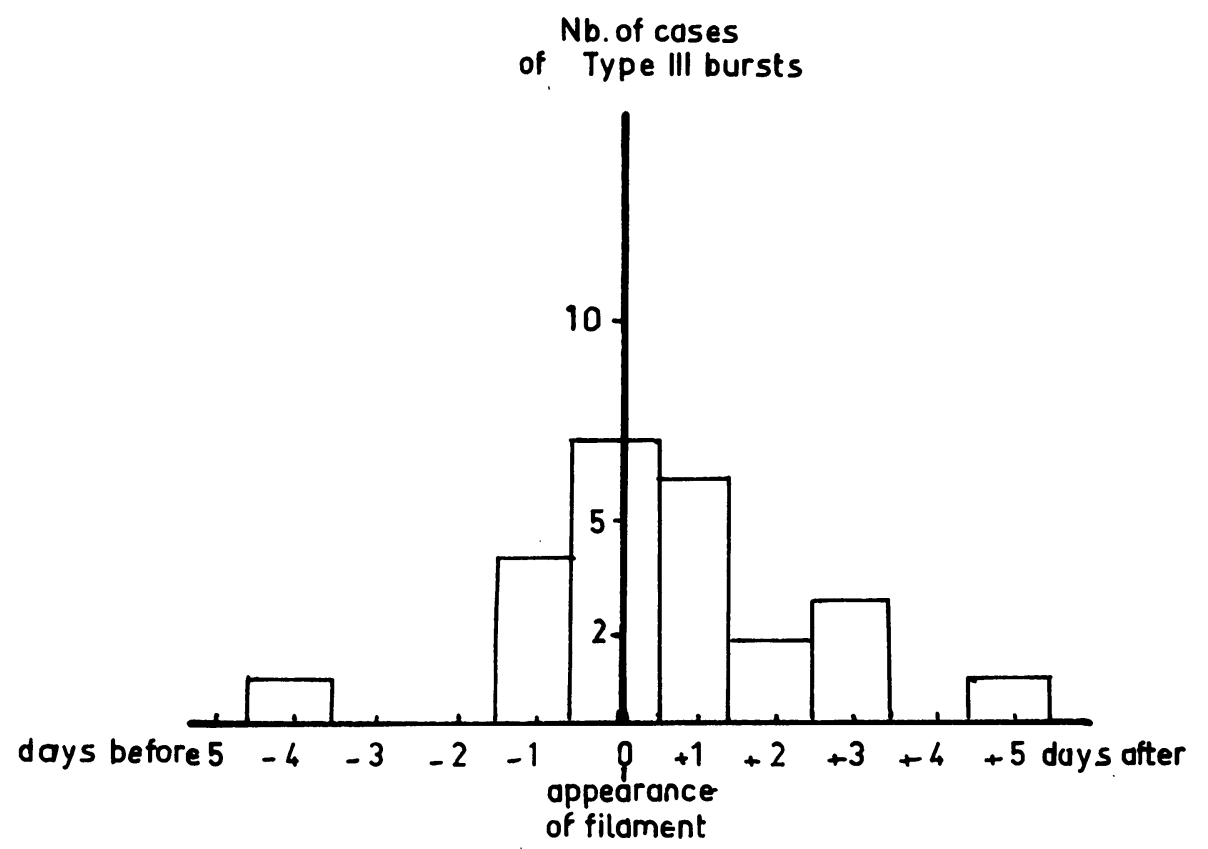

Fig. 3. Histogram of the distribution of delays between the occurrence of type III bursts and the appearance of filaments. Negative delay indicates that the type III bursts occurred before this appearance (from Mercier, 1973).

filament in the order of one day. This fact lead to informations about the condensation process of the prominence.

If it is accepted that type III bursts are generated in coronal neutral sheets, these results would suggest that filaments are condensed from preexisting neutral sheets with a time scale of one day or less. This interpretation is consistent with the theoretical picture given by Kuperus and Raadu in which filaments result on thermal instability in neutral magnetic sheets.

Inside such coronal structures, the electronic density may be estimated by observational radio positions, as is to be discussed by Rosenberg in the present Symposium. It is difficult to reach such information by ground coronometer observations (eclipses apart) due to the extreme location, the fast evolution, the size and the integration along the line of sight.

The observational radio results reveal, with good conditions, the exact root of this structure in the low solar atmosphere. 


\section{References}

Axisa, F.: 1974, Solar Phys. 35, 207-224.

Mercier, C.: 1973, Solar Phys. 33, 177.

\section{DISCUSSION}

Leblanc: What is the position of type III bursts relative to quiescent filaments?

Rosenberg: We studied the III-filament relation from one-dimensional Nancay observations and concluded that filaments certainly do influence the location of type III bursts. This is only a partial answer to your question. 